\title{
A!
}

This is an electronic reprint of the original article.

This reprint may differ from the original in pagination and typographic detail.

Qin, H. J.; Tsurkan, S.; Ernst, A.; Zakeri, Kh

\section{Experimental Realization of Atomic-Scale Magnonic Crystals}

Published in:

Physical Review Letters

DOI:

10.1103/PhysRevLett.123.257202

Published: 18/12/2019

Document Version

Publisher's PDF, also known as Version of record

Please cite the original version:

Qin, H. J., Tsurkan, S., Ernst, A., \& Zakeri, K. (2019). Experimental Realization of Atomic-Scale Magnonic

Crystals. Physical Review Letters, 123(25), [257202]. https://doi.org/10.1103/PhysRevLett.123.257202

This material is protected by copyright and other intellectual property rights, and duplication or sale of all or part of any of the repository collections is not permitted, except that material may be duplicated by you for your research use or educational purposes in electronic or print form. You must obtain permission for any other use. Electronic or print copies may not be offered, whether for sale or otherwise to anyone who is not an authorised user. 


\title{
Experimental Realization of Atomic-Scale Magnonic Crystals
}

\author{
H. J. Qin, ${ }^{1,2}$ S. Tsurkan, ${ }^{1}$ A. Ernst, ${ }^{3,4}$ and Kh. Zakeri॰ ${ }^{1, *}$ \\ ${ }^{1}$ Heisenberg Spin-dynamics Group, Physikalisches Institut, Karlsruhe Institute of Technology, \\ Wolfgang-Gaede-Straße 1, D-76131 Karlsruhe, Germany \\ ${ }^{2}$ NanoSpin, Department of Applied Physics, Aalto University School of Science, FI-00076 Aalto, Finland \\ ${ }^{3}$ Institute for Theoretical Physics, Johannes Kepler University, Altenberger Straße 69, 4040 Linz, Austria \\ ${ }^{4}$ Max-Planck-Institut für Mikrostrukturphysik, Weinberg 2, D-06120 Halle, Germany
}

(Received 5 June 2019; published 18 December 2019)

\begin{abstract}
We introduce a new approach of materials design for terahertz magnonics making use of quantum confinement of terahertz magnons in layered ferromagnets. We show that in atomically designed multilayers composed of alternating atomic layers of ferromagnetic metals one can efficiently excite different magnon modes associated with the quantum confinement in the third dimension, i.e., the direction perpendicular to the layers. We demonstrate experimentally that the magnonic band structure of these systems can be tuned by changing the material combination and the number of atomic layers. We realize the idea of opening band gaps, with a size of up to several tens of millielectronvolts, between different terahertz magnon bands and thereby report on the first step toward the realization of atomic-scale magnonic crystals.
\end{abstract}

DOI: 10.1103/PhysRevLett.123.257202

In solid-state physics a main idea is to utilize the elementary magnetic excitations, magnons, for information processing [1-3]. The realization of this idea would allow energy efficient information processing without Ohmic losses [4]. The magnon spectrum covers a wide range of frequencies from gigahertz up to several terahertz. It has been shown that the dispersion relation of gigahertz magnons in structures with periodically modulated magnetic properties exhibits additional branches [5-16]. The magnonic band structure of such artificially designed structures, called magnonic crystals, exhibits bands of allowed magnon states as well as forbidden gaps, similar to photonic crystals [17]. The concept of magnonic crystals is an important concept in magnonics and spintronics. Since these structures permit the propagation of magnon modes required for specific operations, they serve as platforms for logic devices $[8,16]$. However, so far only device operations in the gigahertz regime have been demonstrated [16].

Another interesting part of the magnon spectrum is the part describing terahertz magnons. These magnons are ultrafast and allow device operations in terahertz frequencies. Unfortunately, the approach mentioned above cannot be used for designing a magnonic crystal capable of operation in such high frequencies. This is due to the fact that for increasing the operation frequency from gigahertz to terahertz one needs to make the structures smaller and smaller. At the same time the type of magnetic interaction, determining the magnon energies, changes while going from large to small length scales. Hence, for designing such structures the relevant properties to modulate are either the short-range magnetic exchange interaction or the atomic spins. Moreover, such modulations should be realized on atomic scales (ultimately on neighboring atoms), which are not practically feasible [18].

In this Letter, we propose an inherently different design strategy, making use of quantum confinement of terahertz magnons in layered structures composed of a few atomic layers. We design synthetic multilayers of 3-5 atomic layers made of two different ferromagnetic materials (Fe and $\mathrm{Co}$ ). We efficiently excite and detect different terahertz magnon modes associated with quantum confinement in the direction perpendicular to the layers. By tuning interatomic exchange parameters, we experimentally realize the engineering of terahertz magnon band structures and opening band gaps up to tens of millielectronvolts between different magnonic bands. Our results provide a new design strategy toward magnonic crystals on atomic length scales and, for the first time, bring the concept of utilizing terahertz quantum confined magnons for application in magnonics into discussion.

Generally, an atomic layer of an elemental ferromagnet shall exhibit a single semiparabolic magnon band, which in the absence of relativistic effects and external magnetic field satisfies the Goldstone theorem [20]. This magnon band is confined in the layer. Now, if one increases the number of atomic layers, one would observe the appearance of additional magnon modes and the magnonic band structure develops to a multiband system. The presence of additional magnon modes is a consequence of quantum confinement in the direction perpendicular to the layers. In a multilayer composed of $N$ atomic layers $N$ magnon modes with quantization numbers $n=0,1, \ldots, N-1$ are expected to form with continuous momentum in the film plane. Only the lowest energy mode $(n=0$ mode $)$ is 


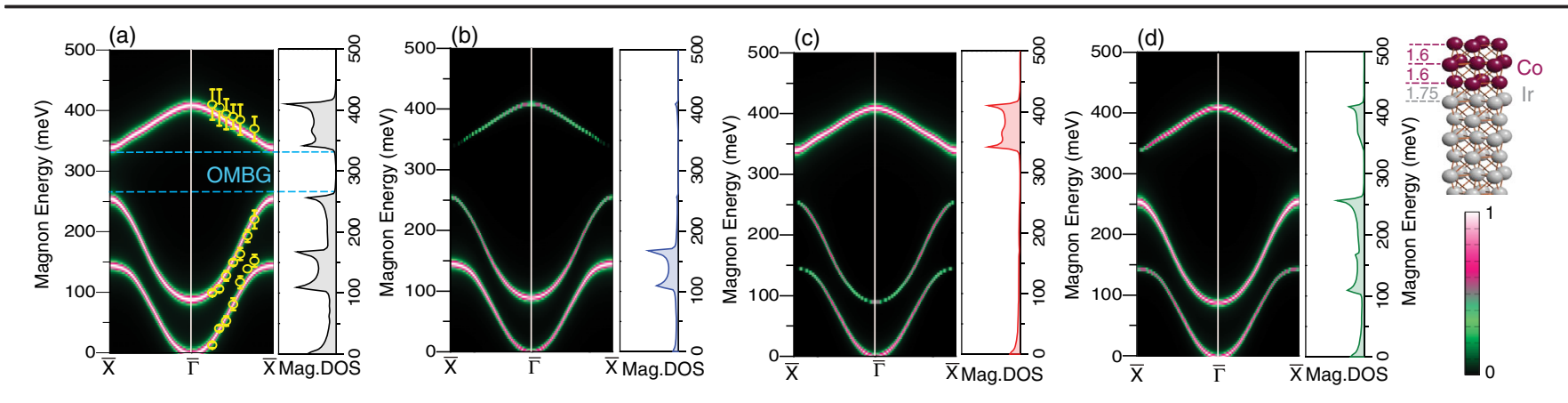

FIG. 1. (a) The first principles magnon Bloch spectral function (BSF) and the magnon density of states (DOS) projected onto all three slabs of the Co film. The experimental magnon dispersion relation is shown by open circles. The horizontal axis represents the magnons' in-plane momentum. The data indicate an "optical magnon band gap" in the energy range of 260-340 meV. The magnon BSF as well as the magnon DOS projected into the interface atomic layer (b), the middle layer (c), and the surface layer (d). The results indicate that the main contribution to the $n=2$ magnon band is coming from the spins located in the middle Co atomic layer, while the main contribution to the $n=0$ and $n=1$ magnon modes is coming from the spins located in the interface and surface layers, respectively. The interlayer distances used for $a b$ initio calculations are given in angstroms.

referred to as the acoustic mode and satisfies the Goldstone theorem. The higher energy modes are the quantum confined magnon modes (sometimes referred to as optical magnons) and have a nonzero energy at the Brillouin zone center. The position and the shape of the magnon bands depend on the strength and the sign of the interatomic exchange parameters which can be tuned by changing the interatomic distances [21-31]. The interatomic distances can be changed by epitaxial growth of the layers on different substrates [32]. Note that although the higher energy magnon modes are the quantum confined magnons, they possess an in-plane momentum and a characteristic dispersion relation and therefore propagate in the film. Experimentally, the dispersion relation of quantized magnon modes in an ultrathin ferromagnetic film composed of a few atomic layers can be probed by spin-polarized highresolution electron energy loss spectroscopy, as has been shown recently [34].

In order to experimentally realize a multimagnon band system with a band gap in the optical magnon region i.e., an "optical magnon band gap" (OMBG), an ultrathin Co film with a thickness of three atomic layers was grown on $\operatorname{Ir}(001)-1 \times 5$. The magnon spectrum was probed by spin-polarized high-resolution electron energy loss spectroscopy with the particular attention to the $n=1$ and $n=$ 2 magnon modes (see Supplemental Material, Sec. I [33] for materials and methods). The results of our analysis are shown as open circles in Fig. 1(a) together with the calculated magnonic band structure, based on first principles, indicating an OMBG of about $80 \mathrm{meV}$ in the energy range of 260-340 meV [32]. The importance of the gap opening, in general, is as follows. If such a system is exposed to a continuous spectrum of terahertz magnonic waves, in the regions of allowed states magnons can be transmitted and be used for further processing. In the regions of the band gaps, however, no magnon transmission takes place. The system would therefore act as a terahertz magnonic platform allowing the propagation of certain terahertz magnons. Similarly, when the system is exposed to a continuous spectrum of an electromagnetic wave, it would act as a terahertz filter or sensor (no absorption occurs by magnons in the frequency regions where the band gaps are located). The position and the width of the band gap would define the functionality of such a filter or sensor.

Our next aim is to open a gap between the $n=0$ and $n=1$ magnon bands, i.e., an "acoustic-optical magnon band gap" (AOMBG). The importance of an AOMBG is twofold. First, it would allow us to take advantage of acoustic magnons. These magnons possess longer lifetimes, are ultrafast, and can propagate on nanometer length scales within a few tens of femtoseconds. They would push the limit of operation speeds and length scales to ultimate values. Second, the physics of an AOMBG opening is of great fundamental interest in the context of topological magnon states in multiband magnon systems as well as in 2D magnets. This would allow for a fundamental understanding of the origin of the gap opening in such complex systems [35]. As it is apparent from Fig. 1(a) a simple scaling of the $n=0$ and $n=1$ bands would not lead to an AOMBG. Therefore, one first needs to know how the bands are formed and where the main contribution to the magnonic band structure comes from. In the viewpoint of spatial localization each magnon mode is mainly localized in a part of the multilayer structure. In the language of classical dynamics the dynamical parts of the spins located in different layers have different contributions to each magnon mode. To reveal the spatial localization of magnons (or the contribution of each atomic layer to a specific magnon mode) one needs to calculate, based on first principles, the so-called Bloch spectral function (BSF) of magnons. The projected BSF onto each layer would reveal the contribution of that specific layer to the magnon mode. This means if a magnon mode is mainly localized at one specific layer, the projected BSF of this mode onto this 
layer should be the largest. BSF is analogous to the spectral function of the Bloch electrons in solids. The integral of BSF over $\mathbf{q}$ shall provide the density of magnonic states. If one calculates the projection of the magnonic density of states (DOS) onto different layers, one would be able to map the spatial localization of the magnons.

Generally, if the surface and interface layers are identical, the $n=0$ and $n=1$ modes will be equally distributed in these two layers. However, the presence of the substrate breaks the symmetry and leads to the fact that the exchange parameters in these two layers are different. This fact results in differences in the layer-resolved BSF. The calculated BSF for three atomic layers of Co grown on $\operatorname{Ir}(001)-1 \times 5$ is presented in Fig. 1. Figure 1(a) represents the projected BSF onto all three layers. The magnon DOS, calculated by integrating BSF along this symmetry direction $(\bar{\Gamma}-\bar{X})$, is also presented. Since in this case all atomic layers are considered, we call this quantity the "total magnon DOS." Figures 1(b)-1(d) show the projected BSF onto the first, second, and third atomic layer, respectively. Likewise, we present the projected magnon DOS on each atomic layer, next to the projected BSF. In this case the magnon DOS can be called "layer-resolved magnon DOS" (LRDOS). For all magnon bands, the perpendicular component of the wave vector is quantized and is given by $q_{\perp}=n \pi / N$. While projecting BSF onto the top or bottom atomic layer [see, e.g., Figs. 1(b) and 1(d)] one observes that the degree of localization changes while moving from the zone center toward the zone boundary. The $n=0$ magnon band becomes more localized in the layer in which the exchange parameters are smaller (the interface layer). The $n=1$ magnon band has a tendency to be mainly localized in the surface layer. The larger the difference in the exchange constants, the more dramatic the spatial localization of magnons. The $n=2$ magnon mode is mainly localized in the middle layer, since this layer is strongly coupled to the top and bottom layer. Hence, the spectral function of this mode has the largest weight when it is projected onto this layer [see Fig. 1(c)]. The same conclusion can be drawn when looking at the LRDOS of magnons. The magnon LRDOS of the interface atomic layer covers mainly the bottom part of the energy spectrum, i.e., the part where the $n=0$ magnon mode exists. The magnon LRDOS of the surface and middle layer covers the middle and the top part of the energy spectrum, respectively, where the $n=1$ and $n=2$ modes are lying. The conclusion of this analysis is that if the aim is to change the $n=0$ mode, one should mainly modify the interface layer [63].

In order to open an $A O M B G$ one should reduce the energy of the $n=0$ mode (by modifying the corresponding atomic layer), keeping the $n=1$ mode unchanged. However, as mentioned above, a simple scaling of the $n=0$ band would not lead to any gap opening. Now, the key idea is to take advantage of competing exchange interactions between nearest and next nearest neighbor spins. This means one should modify the interface layer (the layer which is mainly responsible for this mode) in a specific way. The best way would be to replace this layer with another ferromagnetic layer. The material of choice for the first atomic layer should fulfill three criteria. First of all, the intralayer exchange parameters in the interface layer shall be weak. Second, the next nearest neighbor exchange parameter shall be smaller than the nearest neighbor one.
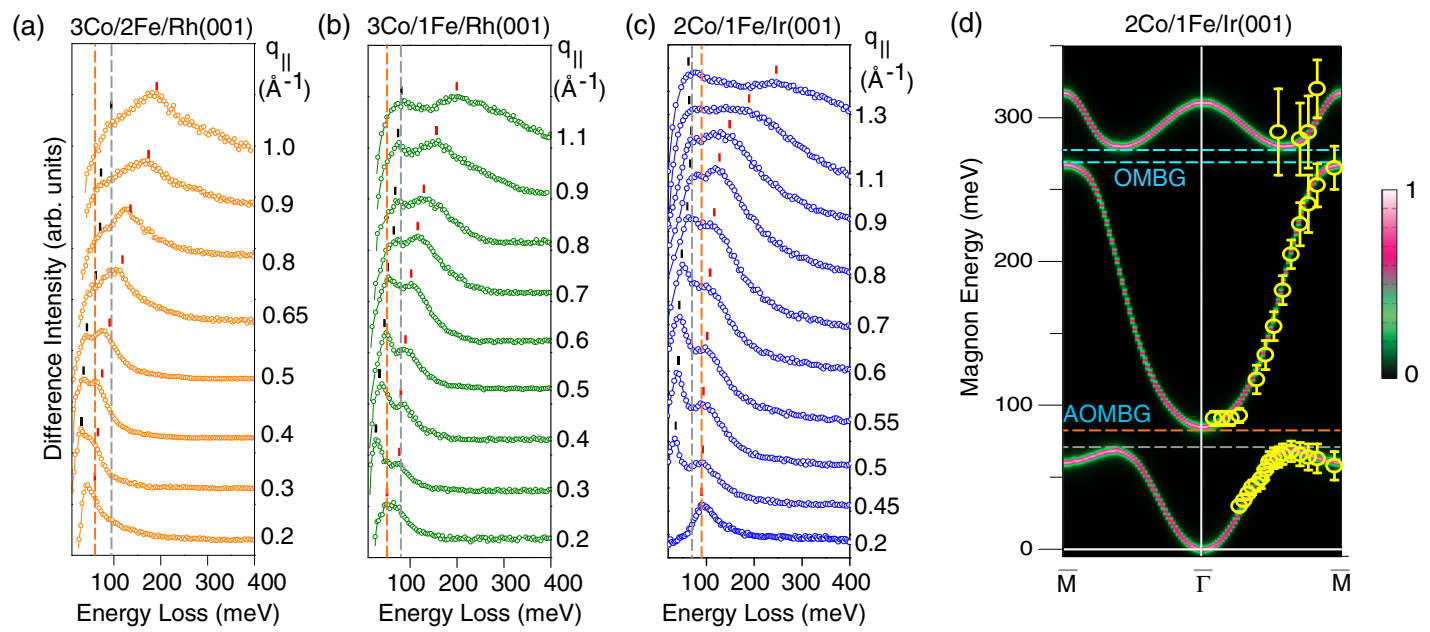

FIG. 2. Realization of an atomic-scale magnonic crystal: The $2 \mathrm{Co} / 1 \mathrm{Fe} / \operatorname{Ir}(001)-1 \times 5$ system. (a), (b), and (c). The experimental difference spectra probed at different in-plane wave vectors along the high symmetry $\bar{\Gamma}-\bar{M}$ direction of the surface Brillouin zone. The gray and orange vertical dashed lines indicate the position of the top and bottom of the $n=0$ and $n=1$ magnon bands, respectively. In the case of $3 \mathrm{Co} / 2 \mathrm{Fe} / \mathrm{Rh}(001)$ and $3 \mathrm{Co} / 1 \mathrm{Fe} / \mathrm{Rh}(001)$ the value of $\mathrm{AOMBG}$ is negative (no band gap is opened). In the case of $2 \mathrm{Co} / 1 \mathrm{Fe} /$ $\operatorname{Ir}(001)-1 \times 5$ an AOMBG of about $25 \mathrm{meV}$ is opened. (d) The magnonic band structure for the $2 \mathrm{Co} / 1 \mathrm{Fe} / \operatorname{Ir}(001)-1 \times 5 \mathrm{system}$. The experimental data are shown by open circles. The results of our modeling are represented as BSF projected onto the whole slabs. 
If the next nearest neighbor exchange parameter is negative it is an advantage, because it would reduce the magnon energies near the zone boundary. Finally, the interlayer exchange interaction should be large enough to push the bottom of the $n=1$ magnon band to higher energies. While the first two criteria can be fulfilled by taking advantage of the interfacial electronic hybridization of an atomic layer of a ferromagnet with a metallic substrate, the final criterion may be fulfilled by tuning the interlayer spacing and taking advantage of tetragonal distortion [32].

For the experimental realization of this idea we systematically design multilayers composed of alternating layers of Fe and Co grown on different substrates, in order to be able to control the magnonic band structure via lattice strain and tetragonal distortion [32]. In Figs. 2(a)-2(c) the difference spectra measured along the high symmetry $\bar{\Gamma}-\bar{M}$ direction for three different multilayer systems, i.e., $3 \mathrm{Co} / 2 \mathrm{Fe} / \mathrm{Rh}(001)$, $3 \mathrm{Co} / 1 \mathrm{Fe} / \mathrm{Rh}(001)$, and $2 \mathrm{Co} / 1 \mathrm{Fe} / \operatorname{Ir}(001)-1 \times 5$ are presented. The energies of both $n=0$ (indicated by the black marker) and $n=1$ (indicated by the red marker) magnon modes increase with increasing $q_{\|}$. In the case of $3 \mathrm{Co} / 2 \mathrm{Fe} / \mathrm{Rh}(001)$ and $3 \mathrm{Co} / 1 \mathrm{Fe} / \mathrm{Rh}(001)$ the energy of the $n=0$ magnon mode exceeds the one of the $n=1$ magnon mode near the zone center $\left[\hbar \omega\left(q_{\|}=0\right)\right]$ and goes even beyond that. The positions of the maximum energy of the $n=0$ mode and the minimum energy of the $n=1$ magnon mode are marked by gray and orange dashed lines, respectively. In the case of $2 \mathrm{Co} / 1 \mathrm{Fe} / \operatorname{Ir}(001)-1 \times 5$ one clearly observes that the energy of the $n=0$ magnon mode never reaches the one of the $n=1$ magnon mode. The magnon band structure of this system is presented in Fig. 2(d). The experimental data are shown by the open symbols. The system is modeled by a Heisenberg model and the results are presented in Fig. 2(d) together with the experimental data (see [33], Sec. I, for materials and methods). The contour plot shows BSF calculated based on this model. The spectral function is projected onto all three atomic layers. One clearly observes an AOMBG of about $25 \mathrm{meV}$. This is the first realization of the atomic-scale magnonic crystals with an AOMBG [64].

We have examined various synthetic multilayers composed of different layers and with different sequences of atomic layers. The results are summarized in Fig. 3. We define the AOMBG as the energy difference between the bottom of the $n=1$ band (at the $\bar{\Gamma}$ point) and the top of the $n=0$ band, i.e., AOMBG $=\hbar \omega_{\bar{\Gamma}}^{n=1}-\hbar \omega_{\max }^{n=0}$. In addition, we also show the values of OMBG, defined as the energy difference between the bottom of the $n=$ $N-1$ band and the top of the $n=N-2$ band i.e., $\mathrm{OMBG}=\hbar \omega_{\min }^{n=N-1}-\hbar \omega_{\bar{X} \text { or } \bar{M}}^{n=N-2}$. These quantities are plotted in Fig. 3 for different synthetic multilayers. $3 \mathrm{Co} / 1 \mathrm{Fe} /$ $\mathrm{Rh}(001)$ shows a very small negative AOMBG, similar to $3 \mathrm{Co} / 1 \mathrm{Fe} / \operatorname{Ir}(001)-1 \times 5$. If one reduces the thickness of Co films from 3 to 2.5 monolayers an AOMBG opens up. Further decreasing the thickness of Co films leads to the

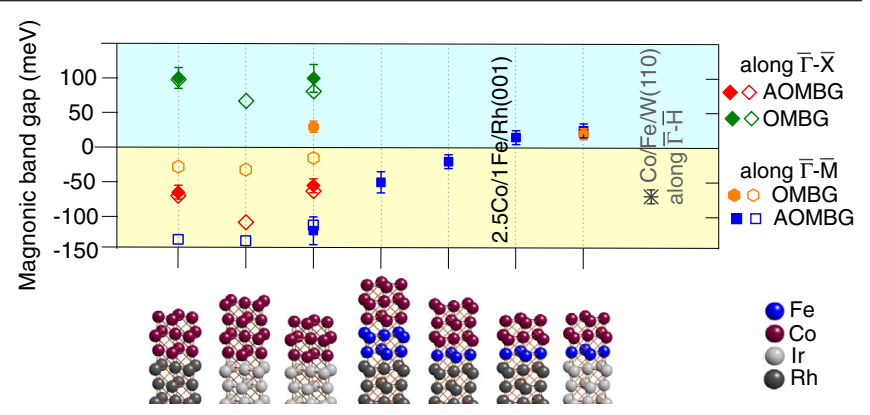

FIG. 3. Magnonic band gaps in different designed layered structures. The measured band gaps are shown by the filled symbols while the results of $a b$ initio calculations are shown by open symbols. The values are shown along two main symmetry directions of the fcc(001) surface Brillouin zone, i.e., $\bar{\Gamma}-\bar{X}$ and $\bar{\Gamma}-\bar{M}$. The energy gap between $n=0$ and $n=1$ magnon modes of $\mathrm{Co} / \mathrm{Fe} / \mathrm{W}(110)$ is shown for a comparison.

formation of a rather complex magnetic ground state for this system. This is owed to the fact that the pattern of the exchange parameters in this system is rather complex [22].

Based on our discovery, we propose a similar design strategy for designing magnonic crystals suitable for operations at subterahertz. Basically, in such a case the atomic layers can be replaced by thin magnetic films. In addition, one may replace the direct Heisenberg exchange with the indirect exchange, known as Ruderman-KittelKasuya-Yosida interaction [65-67]. We suggest that such an idea can be realized by introducing a nonmagnetic spacer layer between the ferromagnetic films. Such multilayer structures shall exhibit magnon energies on the order of subterahertz. If the idea is to construct a three dimensional magnonic structure, the multilayer may be patterned in any desired periodic form, similar to the study performed in Ref. [68].

In summary, we report the first experimental realization of atomic-scale magnonic crystals, capable of operation in the terahertz frequency regime. We demonstrate that in atomically designed ferromagnetic layered structures one can excite multiple magnon modes and, more importantly, open band gaps in different parts of the magnonic band structure. We discuss the key idea behind this band gap opening, being the quantum engineering of the interatomic exchange interaction at the desired part of the structure. We anticipate that our results will be useful for the design of platforms for terahertz magnonics, terahertz sensors, and terahertz electromagnetic filters. Moreover, the physics of magnonic band structure and the origin of $\mathrm{OMBG}$ and AOMBG would be of great interest in the context of topological magnons and 2D magnets [35].

Kh. Z. and S. T. acknowledge funding from the Deutsche Forschungsgemeinschaft (DFG) through the Heisenberg Programme No. ZA 902/3-1 and No. ZA 902/6-1 and the DFG Grant No. ZA 902/4-1. The work of A.E. was supported by the DFG via the Collaborative Research 
Centre 762 (Project No. A4). H. J. Q. acknowledges funding from Academy of Finland (Grant No. 317918). Kh. Z. thanks the Physikalisches Institut for hosting the group and providing the necessary infrastructure.

*khalil.zakeri@partner.kit.edu

[1] A. Khitun, M. Bao, and K. L. Wang, J. Phys. D 43, 264005 (2010).

[2] S. Demokritov and A. Slavin, Magnonics: From Fundamentals to Applications, Topics in Applied Physics (Springer, Berlin \& Heidelberg, 2012).

[3] A. V. Chumak, V. I. Vasyuchka, A. A. Serga, and B. Hillebrands, Nat. Phys. 11, 453 (2015).

[4] Editorial, Nat. Phys. 11, 437 (2015).

[5] J. Bland and B. Heinrich, Ultrathin Magnetic Structures III: Fundamentals of Nanomagnetism, Ultrathin Magnetic Structures (Springer, Berlin \& Heidelberg, 2005).

[6] S. Tacchi, T. N. Anh Nguyen, G. Carlotti, G. Gubbiotti, M. Madami, R. K. Dumas, J. W. Lau, J. Åkerman, A. Rettori, and M. G. Pini, Phys. Rev. B 87, 144426 (2013).

[7] S. Demokritov, Spin Wave Confinement: Propagating Waves, 2nd ed. (Pan Stanford Publishing, New York, 2017).

[8] V. V. Kruglyak, S. O. Demokritov, and D. Grundler, J. Phys. D 43, 264001 (2010).

[9] A. A. Serga, A. V. Chumak, and B. Hillebrands, J. Phys. D 43, 264002 (2010).

[10] B. Lenk, H. Ulrichs, F. Garbs, and M. Münzenberg, Phys. Rep. 507, 107 (2011).

[11] A. V. Chumak, T. Neumann, A. A. Serga, B. Hillebrands, and M. P. Kostylev, J. Phys. D 42, 205005 (2009).

[12] K.-S. Lee, D.-S. Han, and S.-K. Kim, Phys. Rev. Lett. 102, 127202 (2009).

[13] G. Gubbiotti, S. Tacchi, M. Madami, G. Carlotti, A. O. Adeyeye, and M. Kostylev, J. Phys. D 43, 264003 (2010).

[14] S. Tacchi, F. Montoncello, M. Madami, G. Gubbiotti, G. Carlotti, L. Giovannini, R. Zivieri, F. Nizzoli, S. Jain, A. O. Adeyeye et al., Phys. Rev. Lett. 107, 127204 (2011).

[15] M. Vogel, A. V. Chumak, E. H. Waller, T. Langner, V. I. Vasyuchka, B. Hillebrands, and G. von Freymann, Nat. Phys. 11, 487 (2015).

[16] A. V. Chumak, A. A. Serga, and B. Hillebrands, J. Phys. D 50, 244001 (2017).

[17] J. D. Joannopoulos, P. R. Villeneuve, and S. Fan, Nature (London) 386, 143 (1997).

[18] Although modulations on atomic scales already exist in ferrimagnetic materials, such materials exhibit very complex magnonic band structures with no possibility for tuning the bands in a desired way [19].

[19] A. J. Princep, R. A. Ewings, S. Ward, S. Toth, C. Dubs, D. Prabhakaran, and A. T. Boothroyd, npj Quantum Mater. 2, 63 (2017)

[20] The Goldstone theorem implies that it is, in principle, possible to produce long-wavelength excitations (excitations with $q=0$ at the Brillouin zone center) for vanishingly small energy cost. Note that the semiparabolic behavior is only valid for the region of small wave vectors $q \rightarrow 0$.
[21] K. Zakeri, T.-H. Chuang, A. Ernst, L. Sandratskii, P. Buczek, H. Qin, Y. Zhang, and J. Kirschner, Nat. Nanotechnol. 8, 853 (2013).

[22] Y. Meng, K. Zakeri, A. Ernst, T.-H. Chuang, H. J. Qin, Y.-J. Chen, and J. Kirschner, Phys. Rev. B 90, 174437 (2014).

[23] T.-H. Chuang, K. Zakeri, A. Ernst, L. M. Sandratskii, P. Buczek, Y. Zhang, H. J. Qin, W. Adeagbo, W. Hergert, and J. Kirschner, Phys. Rev. Lett. 109, 207201 (2012).

[24] K. Zakeri, Phys. Rep. 545, 47 (2014).

[25] K. Zakeri, J. Phys. Condens. Matter 29, 013001 (2017).

[26] T.-H. Chuang, K. Zakeri, A. Ernst, Y. Zhang, H. J. Qin, Y. Meng, Y.-J. Chen, and J. Kirschner, Phys. Rev. B 89, 174404 (2014).

[27] J. Prokop, W. X. Tang, Y. Zhang, I. Tudosa, T. R. F. Peixoto, K. Zakeri, and J. Kirschner, Phys. Rev. Lett. 102, 177206 (2009).

[28] A. Bergman, A. Taroni, L. Bergqvist, J. Hellsvik, B. Hjörvarsson, and O. Eriksson, Phys. Rev. B 81, 144416 (2010).

[29] A. Taroni, A. Bergman, L. Bergqvist, J. Hellsvik, and O. Eriksson, Phys. Rev. Lett. 107, 037202 (2011).

[30] L. Bergqvist, A. Taroni, A. Bergman, C. Etz, and O. Eriksson, Phys. Rev. B 87, 144401 (2013).

[31] O. Eriksson, A. Bergman, L. Bergqvist, and J. Hellsvik, Atomistic Spin Dynamics: Foundations and Applications (Oxford University Press, Oxford, 2016).

[32] The position and the value of this band gap can be tuned by changing the number of $\mathrm{Co}$ atomic layers, tuning the interlayer spacing and taking advantage of tetragonal distortion. For an illustration of this idea please refer to Sec. II of [33].

[33] See Supplemental Material at http://link.aps.org/ supplemental/10.1103/PhysRevLett.123.257202 for extended discussions, which includes Refs. [20-30,34-62].

[34] Y.-J. Chen, K. Zakeri, A. Ernst, H. J. Qin, Y. Meng, and J. Kirschner, Phys. Rev. Lett. 119, 267201 (2017).

[35] D. L. Cortie, G. L. Causer, K. C. Rule, H. Fritzsche, W. Kreuzpaintner, and F. Klose, Adv. Funct. Mater. 0, 1901414 (2019).

[36] K. Zakeri, T. Peixoto, Y. Zhang, J. Prokop, and J. Kirschner, Surf. Sci. 604, L1 (2010).

[37] R. Vollmer, M. Etzkorn, P. S. Anil Kumar, H. Ibach, and J. Kirschner, Phys. Rev. Lett. 91, 147201 (2003).

[38] M. Etzkorn, P. S. Anil Kumar, W. Tang, Y. Zhang, and J. Kirschner, Phys. Rev. B 72, 184420 (2005).

[39] W. X. Tang, Y. Zhang, I. Tudosa, J. Prokop, M. Etzkorn, and J. Kirschner, Phys. Rev. Lett. 99, 087202 (2007).

[40] K. Zakeri, Y. Zhang, J. Prokop, T.-H. Chuang, N. Sakr, W. X. Tang, and J. Kirschner, Phys. Rev. Lett. 104, 137203 (2010).

[41] Y. Zhang, P. A. Ignatiev, J. Prokop, I. Tudosa, K. Zakeri, V. S. Stepanyuk, J. Kirschner, T. R. F. Peixoto, and W. X. Tang, Phys. Rev. Lett. 106, 127201 (2011).

[42] K. Zakeri and J. Kirschner, Probing Magnons by SpinPolarized Electrons, Topics in Applied Physics Magnonics from Fundamentals to Applications Vol. 125 (Springer, Berlin, Heidelberg, 2013), pp. 84-99.

[43] Y. Zhang, T.-H. Chuang, K. Zakeri, and J. Kirschner, Phys. Rev. Lett. 109, 087203 (2012). 
[44] K. Zakeri, Y. Zhang, T.-H. Chuang, and J. Kirschner, Phys. Rev. Lett. 108, 197205 (2012).

[45] J. P. Perdew, K. Burke, and M. Ernzerhof, Phys. Rev. Lett. 77, 3865 (1996).

[46] A. I. Liechtenstein, M. I. Katsnelson, V. P. Antropov, and V. A. Gubanov, J. Magn. Magn. Mater. 67, 65 (1987).

[47] P. Soven, Phys. Rev. 156, 809 (1967).

[48] B. L. Gyorffy, Phys. Rev. B 5, 2382 (1972).

[49] K. Heinz and L. Hammer, Prog. Surf. Sci. 84, 2 (2009).

[50] A. T. Costa, R. B. Muniz, and D. L. Mills, Phys. Rev. B 69, 064413 (2004).

[51] H. J. Qin, K. Zakeri, A. Ernst, T.-H. Chuang, Y.-J. Chen, Y. Meng, and J. Kirschner, Phys. Rev. B 88, 020404(R) (2013).

[52] T. Balashov, P. Buczek, L. Sandratskii, A. Ernst, and W. Wulfhekel, J. Phys. Condens. Matter 26, 394007 (2014).

[53] J. Sánchez-Barriga, J. Minár, J. Braun, A. Varykhalov, V. Boni, I. Di Marco, O. Rader, V. Bellini, F. Manghi, H. Ebert et al., Phys. Rev. B 82, 104414 (2010).

[54] J. Sánchez-Barriga, J. Braun, J. Minár, I. Di Marco, A. Varykhalov, O. Rader, V. Boni, V. Bellini, F. Manghi, H. Ebert et al., Phys. Rev. B 85, 205109 (2012).

[55] S. Monastra, F. Manghi, C. A. Rozzi, C. Arcangeli, E. Wetli, H.-J. Neff, T. Greber, and J. Osterwalder, Phys. Rev. Lett. 88, 236402 (2002).

[56] S. P. Bayrakci, D. A. Tennant, P. Leininger, T. Keller, M. C. R. Gibson, S. D. Wilson, R. J. Birgeneau, and B. Keimer, Phys. Rev. Lett. 111, 017204 (2013).

[57] P. Buczek, A. Ernst, and L. M. Sandratskii, Phys. Rev. B 84, 174418 (2011).
[58] P. Buczek, A. Ernst, and L. M. Sandratskii, Phys. Rev. Lett. 106, 157204 (2011).

[59] H. J. Qin, K. Zakeri, A. Ernst, L. M. Sandratskii, P. Buczek, A. Marmodoro, T. H. Chuang, Y. Zhang, and J. Kirschner, Nat. Commun. 6, 6126 (2015).

[60] H. J. Qin, K. Zakeri, A. Ernst, and J. Kirschner, Phys. Rev. Lett. 118, 127203 (2017).

[61] K. Zakeri, J. Prokop, Y. Zhang, and J. Kirschner, Surf. Sci. 630, 311 (2014).

[62] J. Rajeswari, H. Ibach, and C. M. Schneider, Phys. Rev. Lett. 112, 127202 (2014).

[63] Consequently, for changing the $n=1$ and 2 modes one should change mainly the surface and middle layer, respectively.

[64] Since the intralayer exchange parameters in the Fe atomic layer are very weak, it is rather straightforward to imagine that the $n=0$ band is mainly localized in the $\mathrm{Fe}$ layer. Consequently, the $n=1$ band is mainly localized in the surface Co layer.

[65] M. A. Ruderman and C. Kittel, Phys. Rev. 96, 99 (1954).

[66] T. Kasuya, Prog. Theor. Phys. 16, 45 (1956).

[67] K. Yosida, Phys. Rev. 106, 893 (1957).

[68] S. Pal, J. W. Klos, K. Das, O. Hellwig, P. Gruszecki, M. Krawczyk, and A. Barman, Appl. Phys. Lett. 105, 162408 (2014). Note that in this study the sample is not treated as a multilayer composed of separate ferromagnetic layers and the exchange coupling between these layers is not considered. Hence, one should not expect to observe $\mathrm{THz}$ magnons. 